\title{
Plasmid Production
}

National Cancer Institute

\section{Source}

National Cancer Institute. Plasmid Production. NCI Thesaurus. Code C113046.

The production of DNA plasmids. 